\title{
CLASSIFICATION OF AURORAL PRECIPITATION FLUXES BY CHARACTERISTIC PARAMETERS AND THEIR EFFECTS ON THE COUPLING OF THE PRECIPITATION TO THE AMBIENT IONOSPHERE
}

\author{
E. G. Fontheim, ${ }^{*}$ S. F. Fung** and J. D. Winningham*** \\ *Space Physics Research Laboratory, The University of Michigan, Ann Arbor, \\ MI 48109-2143, U.S.A. \\ ** ORI, Inc., Landover, MD 20785, U.S.A. \\ *** Southwest Research Institute, San Antonio, TX 78284, U.S.A.

\section{ABSTRACT}

The spectral shapes of the precipitating auroral electron flux spectra are quantified by an automated fitting procedure which represents each flux spectrum as a superposition of Maxwellian and Gaussian partial fluxes. This makes it possible to represent each spectrum by a set of characteristic parameters which describe the shape of that spectrum. A set of inverted-V events observed by the Low-Energy Plasma Instrument on Dynamics Explorer 2 near the fall 1981 equinox, has been analyzed. The distribution of the peak inverted- $V$ energies in magnetic local time and invariant latitude has been obtained, and it is shown that by far the highest peak energies occur in the range $65<$ II $<70$ and $18<$ MLT $<24$ hours. It is also shown how the difference in spectral characteristics of the precipitation fluxes between the cusp and the nighttime auroral zone determines the thermal coupling of the precipitation to the ambient ionosphere.

\section{DESCRIPTION OF FITTING PROCEDURE}

In a recent paper, Fontheim et al. $/ 1 /$ described an automated fitting program which fits measured auroral precipitation fluxes to a superposition of various Maxwellian and Gaussian partial fluxes. The program was developed with data from the Low Energy Plasma Instrument (LAPI) $/ 2 /$ on Dynamics Explorer 2, a polar orbiting satellite with perigee of $300 \mathrm{~km}$ and apogee of $1100 \mathrm{~km}$. The fitting function used by these authors is

$$
\Phi=\sum_{i}^{5} \frac{M_{i} E}{T_{i}^{3 / 2}} \exp \left(-\frac{E}{T_{i}}\right)+\sum_{1}^{4} F_{i} E \exp \left(-\left(\frac{E-E_{o i}}{\Delta_{i}}\right)^{2}\right)
$$

where $\mathrm{M}_{\mathrm{i}}, \mathrm{T}_{\mathrm{i}}, \mathrm{F}_{\mathrm{i}}, \mathrm{E}_{\mathrm{oi}}$, and $\Delta_{\mathrm{i}}$ are the fitting parameters, and the temperatures $T_{\mathrm{i}}$ are in energy units. The indices "i" in the above expression order the Maxwellian and Gaussian functions in increasing values of the parameters $T_{i}$ and $E_{0 i}$, respectively. The sums do not represent piecewise fits but true superpositions over the entire energy range (in this case $5 \mathrm{eV}<\mathrm{E}<27 \mathrm{keV}$ ). The number of Maxwellian and Gaussian partial fluxes used in a particular fit is determined by a criterion minimizing the fitting error. In most cases two or three Maxwellians lead to a fit of sufficient accuracy. Any higher order Maxwellians represent energetic tails of the flux spectra. The Gaussian partial fluxes represent humps on the tail of the fitted flux. Those fluxes which have no humps, obviously have no Gaussian contributions. The program allows for up to four humps. Fig. 1 shows an example of a fitted flux spectrum with two humps.

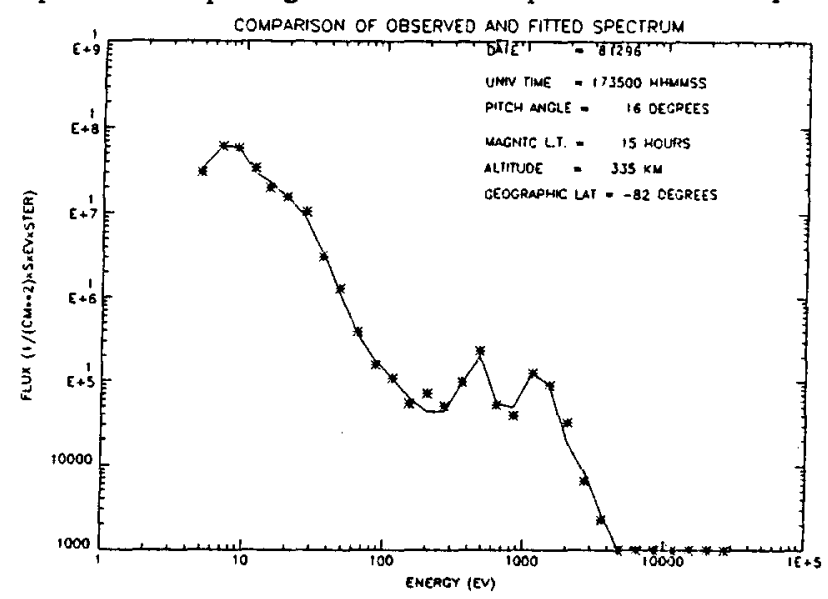

Fig. 1. Comparison of a sample observed spectrum with a fitted spectrum. The asterisks represent the measured values and the curve the fitted spectrum. The spectrum was fitted to a sum of three Maxwellian partial fluxes with temperatures of $7.45 \mathrm{eV}, 33.5 \mathrm{eV}$, and $634 \mathrm{eV}$, respectively, and two Gaussian fluxes with peak energies of $459 \mathrm{eV}$ and $1320 \mathrm{eV}$, respectively. 
The fitting is carried out automatically, i.e., data are inputted from some source (usually a tape), the program runs in batch mode, and the output is stored in a file together with the corresponding orbital data, certain magnetic indices, and several error and significance parameters for each spectrum. The set of fitting parameters (the $\mathrm{M}_{\mathrm{i}}, \mathrm{T}_{\mathrm{i}}, \mathrm{F}_{\mathrm{i}}, \mathrm{E}_{\mathrm{oi}}, \Delta_{\mathrm{i}}$ ) uniquely describes a given flux spectrum and is completely equivalent to the representation of the spectrum in terms of the flux values at given energies. The advantage of the parameter description is, however, that it represents a digital description of the characteristic features of the spectrum and therefore lends itself to investigations of physical processes which involve the spectral shapes of the fluxes. A large number of such parameter sets are being stored in a data base. This data base can be manipulated and searched without the necessity for some person to have to go through a vast amount of data "by hand". Such a data base makes it possible to carry out a variety of correlative studies among the parameters, or between these parameters and other measurement data, or to investigate the distribution of a certain parameter over a spatial coordinate of interest, such as pitch angle.

\section{PARAMETER VARIATIONS IN PITCH ANGLE AND IN LATITUDE AND MAGNETIC LOCAL TIME}

The present study is based on a set of 106 inverted-V events measured by LAPI at and near the 1981 fall equinox. The same data set was the subject of an earlier study dealing with secondary electrons $/ 3 /$. All of the fluxes in those 106 events have been analyzed by our program and the fitting parameters stored in a data base together with the orbital data and magnetic indices. Figs. 2, 3, and 4 show the pitch angle variation of the peak energies $E_{1}$ and the temperatures $T_{1}$ of three selected spectra. The peak energies show a typical loss cone distribution which is the case in practically all spectra. (The very low $E_{1}$-value slightly above $40^{\circ}$ in Fig. 2 represents a fluctuation of unknown origin). The pitch angle distribution of the temperature $T_{1}$, on the other hand, is not uniform at all. In the examples shown here the distribution shows a maximum near $90^{\circ}$ in Fig. 2 and a very different shape with a minimum near $40^{\circ}$ in Figs. 3 and 4. The cause for these differences is not yet understood.

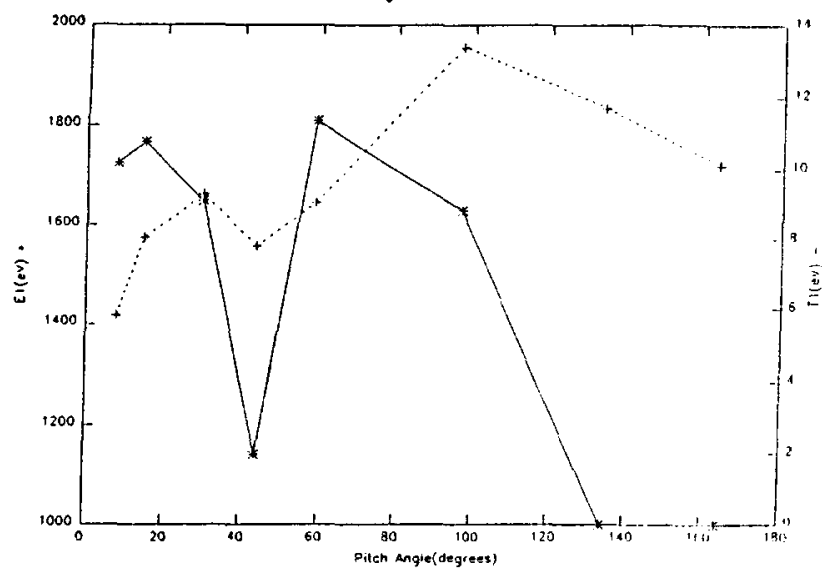
Fig. 2. Pitch angle distributions of $E_{1}$ (solid line) and $T_{1}$ (dotted line) of the electron precipitation spectrum on day
81303 at $04: 14: 23$

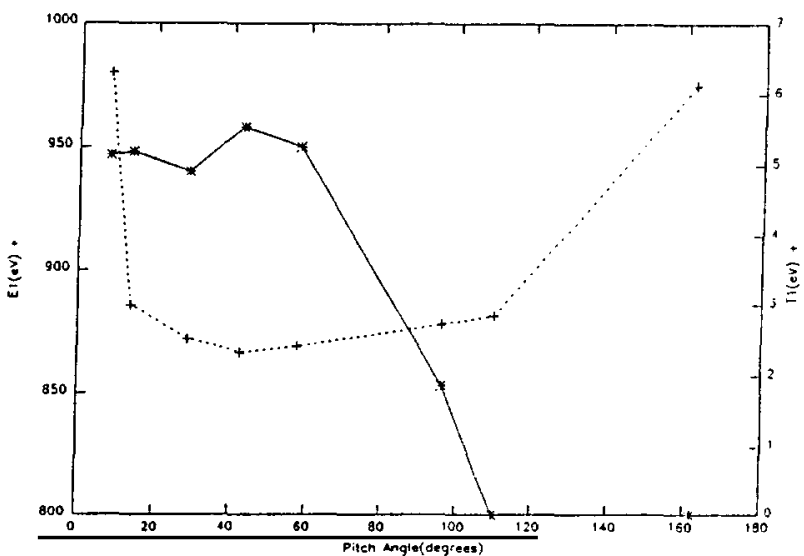

Fig. 3. Pitch angle distributions of $E_{1}$ (solid line) and $T_{1}$ (dotted line) of the electron precipitation spectrum on day 81305 at 23:13:00

The data base of fitting parameters also enables us to determine the distribution of peak energies in invariant latitude and magnetic local time which is presented in Table 1. The table shows that the highest peak energies occur in the invariant latitude range from $65^{\circ}$ to $70^{\circ}$ and in the 18 to $24 \mathrm{MLT}$ sector. The inverted-V events are associated with discrete auroras and originate in all likelihood in the boundary plasma sheet with the near-monoenergetic peaks being generated by some acceleration mechanism somewhere between 1 and 2 earth radii in altitude. Field-aligned electric fields have been identified in many cases as the responsible acceleration mechanism. 


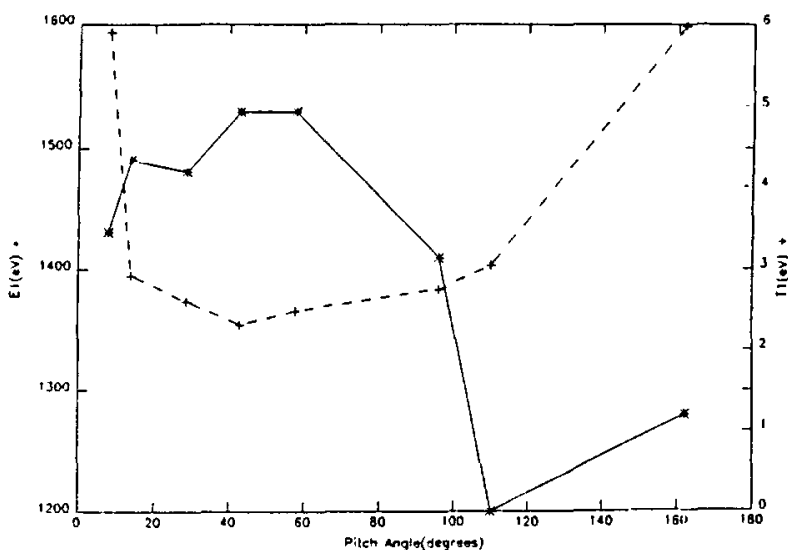

Fig. 4. Pitch angle distributions of $E_{1}$ (solid line) and $T_{1}$ (dotted line) of the electron precipitation spectrum on day 81305 at 23:13:01

$\underline{\underline{T A B L E} 1}$ Distribution of Inverted-V Peak Energies

(Cell Averages of Peak Energies in $\mathrm{eV}$ )

\begin{tabular}{lccc}
\hline INVL & & MLT & \\
& $15-18$ & $18-21$ & $21-24$ \\
$65-70$ & & 7638 & 6101 \\
$70-75$ & 3800 & 3451 & 3515 \\
$75-80$ & & 820 & \\
$80-85$ & & 534 & \\
\hline
\end{tabular}

\section{IMPLICATIONS FOR HEAT INPUT RATES}

Fontheim et al. / $/$ have shown that the intensity $\mathrm{M}_{1}$ of the lowest temperature Maxwellian partial flux (see equation (1)) was considerably higher during a set of stormtime, high-temperature cleft events than during a set of quiettime cleft events. Since the heat input rate by an electron into an electron gas is inversely proportional to the energy of the incident electron, the significance of the intensity $M_{1}$ is that it represents a measure of the heat input rate by the precipitation flux into the ambient electron gas by Coulomb collisions.

TABLE2 Comparison of Fitting Parameter M1 Distributions $1 \mathrm{eV}<\mathrm{T} 1<10 \mathrm{eV}$

\begin{tabular}{|c|c|c|c|c|}
\hline \multirow[b]{2}{*}{ M1 } & \multicolumn{2}{|c|}{ Cusp } & \multicolumn{2}{|c|}{$\begin{array}{l}\text { Evening and Nighttime } \\
\text { Auroral Zone }\end{array}$} \\
\hline & Storm & Quiet & Storm & Quiet \\
\hline $\begin{array}{l}5 \times 10^{* *} 4-5 \times 10^{* * 5} \\
5 \times 10^{* * 5}-5 \times 10^{* * 6} \\
5 \times 10^{* * 6}-5 \times 10^{* * 7} \\
5 \times 10^{* * 7}-5 \times 10^{* * 8} \\
5 \times 10^{* * 8}-5 \times 10^{* * 9} \\
5 \times 10^{* * 9}-5 \times 10^{* * 10}\end{array}$ & $\begin{array}{c}0 \\
0 \\
4.6 \\
27.5 \\
36.8 \\
18.3\end{array}$ & $\begin{array}{l}0 \\
6.4 \\
75.0 \\
5.8 \\
1.6 \\
0\end{array}$ & $\begin{array}{l}10.3 \\
49.7 \\
20.8 \\
0.5 \\
0 \\
0\end{array}$ & $\begin{array}{l}13.2 \\
51.2 \\
20.2 \\
0.4 \\
0.06 \\
0\end{array}$ \\
\hline
\end{tabular}

Distribution is in \%. M1 is in units of $\mathrm{cm}^{-2} \mathrm{~s}^{-1} \mathrm{eV}-0.5$.

A similar comparison has been made between the stormtime and quiettime inverted- $V$ data used in this present study of the evening and nighttime auroral zone. The results are summarized in Table 2 . It is clearly seen that in the vast majority of cleft cases, $M_{1}$ is one to two orders of magnitude higher during stormtime conditions than it is during quietrime conditions. This is at least indirect evidence that Coulomb collisions between precipitating and ambient electrons are an important (if not the dominant) mechanism in heating the ambient electron gas during stormtime conditions in the cleft. This conclusion has been reinforced by a comparison of collisional heat input rates of the stormtime and quiettime cases $/ 1$. The inverted-V events studied in the present paper have been divided into two sets according to their $K_{p}$ values, one set with $K_{p}<2.5$ and the other with $K_{p}>2.5$. The average ambient electron temperature during the quiettime cases $\left(\mathrm{K}_{\mathrm{p}}<2.5\right)$ was $2168^{\circ} \mathrm{K}$ with a standard deviation of $546^{\circ} \mathrm{K}$ and for the stormtime cases $\left(\mathrm{K}_{\mathrm{p}}>2.5\right) 2333^{\circ} \mathrm{K}$ with a standard deviation of $627^{\circ} \mathrm{K}$. The electron temperatures were obtained by the Langmuir probe on Dynamics Explorer $2 / 4 /$ simultaneously with the LAPI particle data. Although the average 
temperature during stormume events is slightly higher than during quiettime events, this difference is not significant in view of the size of the standard deviations. Table 2 shows that there is no significant difference between the stormtime and quiettime distributions of $M_{1}$ for the auroral inverted-V fluxes. In the case of the latter fluxes it should be understood that $M_{1}$ represents the intensity of the low-energy partial flux $\left(T_{1}<10 \mathrm{eV}\right)$ of the spectra which have an "inverted-V hump" at higher energies. This means that the intensities of the lowest temperature Maxwellian components are approximately the same in both cases and so are therefore the collisional heat input rates, provided the ambient electron densities are close to each other in the two cases. The average electron density during the quiettime events was $2 \times 10^{5} \mathrm{~cm}^{-3}$ with a standard deviation of $2 \times 10^{5}$ and that during the stormtime events was $1.4 \times 10^{5} \mathrm{~cm}^{-3}$ with a standard deviation of $0.8 \times 10^{5} / 4 \%$. Hence, there is no significant difference between the two averages. As a result, the average electron temperatures are nearly equal in the two cases.

\section{ACKNOWLEDGMENTS}

The work at the University of Michigan was supported by the National Aeronautics and Space Administration under grants NGR 23-005-015 and NAG 5-472 and by the National Science Foundation under grant ATM 8508753. The work at the Southwest Research Institute was supported by the National Aeronautics and Space Administration under grant NAS 5-28712. The authors are indebted to Eszter Gombosi for carrying out the statistical analysis.

\section{REFERENCES}

1. E.G. Fontheim, L.H. Brace, and J.D. Winningham, Properties of low-energy electron precipitation in the cleft during periods of unusually high ambient electron temperatures, L. Geophys. Res. 92, 12267, (1987)

2. J.D. Winningham, J.L. Burch, N. Eaker, V.A. Blevins, and R.A. Hoffman, The low altitude plasma instrument (LAPI), Space Sci. Instrum. 5, 465, (1981)

3. S.F. Fung and R.A. Hoffman, On the spectrum of the secondary auroral electrons, J. Geophys. Res. 93, 2715 , (1988)

4. L.H. Brace, private communication (1988) 